\title{
Predicting the Frequency Dispersion of Electronic Hyperpolarizabilities on the Basis of Absorption Data and Thomas-Kuhn Sum Rules
}

\author{
Xiangqian Hu, ${ }^{\dagger}$ Dequan Xiao, ${ }^{\dagger}$ Shahar Keinan, ${ }^{\dagger}$ Inge Asselberghs, ${ }^{\dagger}$ Michael J. Therien, ${ }^{*}, \dagger$ \\ Koen Clays, ${ }^{*, *}$ Weitao Yang, ${ }^{*}, \dagger$ and David N. Beratan*, $*, \$$ \\ Department of Chemistry, Duke University, Durham, North Carolina 27708, Department of Chemistry, \\ University of Leuven, Celestijnenlaan 200D, B-3001 Leuven, Belgium, and Department of Biochemistry, \\ Duke University, Durham, North Carolina 27708
}

Received: December 5, 2009

\begin{abstract}
Successfully predicting the frequency dispersion of electronic hyperpolarizabilities is an unresolved challenge in materials science and electronic structure theory. We show that the generalized Thomas-Kuhn sum rules, combined with linear absorption data and measured hyperpolarizability at one or two frequencies, may be used to predict the entire frequency-dependent electronic hyperpolarizability spectrum. This treatment includes two- and three-level contributions that arise from the lowest two or three excited electronic state manifolds, enabling us to describe the unusual observed frequency dispersion of the dynamic hyperpolarizability in high oscillator strength M-PZn chromophores, where (porphinato)zinc(II) (PZn) and metal(II)polypyridyl (M) units are connected via an ethyne unit that aligns the high oscillator strength transition dipoles of these components in a head-to-tail arrangement. We show that some of these structures can possess very similar linear absorption spectra yet manifest dramatically different frequency-dependent hyperpolarizabilities, because of three-level contributions that result from excited state-to-excited state transition dipoles among charge polarized states. Importantly, this approach provides a quantitative scheme to use linear optical absorption spectra and very limited individual hyperpolarizability measurements to predict the entire frequency-dependent nonlinear optical response.
\end{abstract}

\section{Introduction}

Organic optoelectronic materials and devices with enhanced electronic hyperpolarizabilities continue to motivate intensive experimental and theoretical study. ${ }^{1-17}$ Here, our focus is on the electronic first hyperpolarizabilities ( $\beta$ values) of molecules (we refer to this response as the "hyperpolarizability"). "Push-pull" donor-bridge-acceptor motifs often display large hyperpolarizabilities. ${ }^{18-27}$ Many of these chromophores that feature large dynamic hyperpolarizabilities $\left(\beta_{\lambda}\right.$ values) exploit a highly polarizable porphyrinic component. ${ }^{9,17,19,20,23,24,26-37} \mathrm{~A}$ particularly interesting subset of these porphyrin-based high $\beta_{\lambda}$ supermolecules (M-PZn species) feature (porphinato)zinc(II) (PZn) and metal(II)polypyridyl (M) units connected via an ethyne bridge that effectively mixes the PZn $\pi-\pi^{*}$ and metal polypyridyl-based charge-resonance absorption oscillator strength and aligns the respective charge transfer (CT) transition dipoles of these building blocks along the highly conjugated molecular axis. These structures generate extensive interpigment electronic interactions, display unusual dependence of the sign and magnitude of hyperpolarizability on incident irradiation frequency, and manifest extraordinarily large $\beta_{\lambda}$ values at telecommunication relevant wavelengths. ${ }^{9,17,24,28}$ Establishing a framework to understand and to predict these hyperpolarizability dispersion effects is a central challenge in the field of nonlinear optics; this framework could contribute to the further design and optimization of specific device structures. These challenges

* To whom correspondence should be addressed. E-mail: michael.therien@duke.edu (M.J.T.); koen.clays@fys.kuleuven.be (K.C.); weitao.yang@duke.edu (W.Y.); david.beratan@duke.edu (D.N.B.).

${ }^{\dagger}$ Department of Chemistry, Duke University.

* University of Leuven.

${ }^{\S}$ Department of Biochemistry, Duke University. are underscored by the facts that: (i) nonlinear optical (NLO) devices operate at finite wavelengths, so long-wavelength response values (often denoted $\beta_{0}$ ) are of limited practical use and (ii) evaluated $\beta_{0}$ values may not provide direct links to experimental chromophore structure, spectra and (frequency dependent) response.

Quantum chemical analysis ${ }^{23,38-47}$ is used routinely to interpret NLO properties and to assist in molecular design. Yet, developing reliable methods to make quantitative predictions of the frequency dependent $\beta$ spectrum remains an open challenge. ${ }^{48}$ As shown in Table S1 of Supporting Information, ab initio theoretical approaches fail to reproduce experimentally measured $\beta_{\lambda}$ values for large $\pi$-conjugated donor-(porphinato)zincacceptor molecules. A bottleneck toward predicting $\beta_{\lambda}$ is to obtain an accurate description of many electronic excited states that contribute to the hyperpolarizability. The sensitivity of excited state energies and transition moments to basis sets, solvation models, and level of theory is particularly problematic (see Figure S1 of Supporting Information, for example). Experimental studies ${ }^{19,24,49}$ have interpreted $\beta_{\lambda}$ qualitatively using the approximate two-state model (eq 4), with its one- and twophoton resonant enhancement terms, based on the positions of measured linear absorption bands.

Linear absorption spectra provide excitation energies and oscillator strengths. Such extracted information has been successfully used to predict the two photon absorption (2PA) cross section in the lowest-energy dipole-allowed transition based on the customary expression for 2PA obtained from perturbation theory. ${ }^{50}$ Yet, linear spectra do not suffice to predict the hyperpolarizability, since these spectra lack excited state-toexcited state transition dipole moment information required to compute the three-level contributions to $\beta_{\lambda}$. With the aid of 
electroabsorption spectroscopy, Therien and Hupp ${ }^{19}$ identified state-specific contributions (two-level contributions) to the hyperpolarizability from the observed electronic and vibronic transitions for an archetypal porphyrin-based large $\beta_{\lambda}$ chromophore (D-PZn-A) at a very low temperature. M-PZn chromophores such as Ru-PZn and Os-PZn, however, manifest more complex nonlinear responses because of the coupling among the electronic transitions of the building blocks (threelevel contributions). ${ }^{24}$ These studies indicated how CT transitions with distinct transition dipole directions could influence the frequency dispersion of the hyperpolarizability. ${ }^{24}$ Because excited state-to-excited state transition dipoles play a prominent role in determining the NLO response, a qualitative understanding by two-level analysis of $\beta_{\lambda}$ is not sufficient to interpret and predict the frequency dispersion of $\beta_{\lambda}$.

If theory could use information encoded in the linearabsorption spectra to predict the frequency dependent hyperpolarizabilities, especially including three-level contributions (see below), the process of molecular design could be accelerated. Here, we focus on achieving this compelling goal.

The sum-over-states (SOS) expression for hyperpolarizability is ${ }^{51}$

$$
\begin{aligned}
& \beta_{i j k}\left(\omega_{\sigma} ; \omega_{1}, \omega_{2}\right)= \\
& \quad-\sum_{P} \sum_{m, n}\left\{\frac{\langle 0|i| m\rangle\langle m|\tilde{j}| n\rangle\langle n|k| 0\rangle}{\left(E_{m}-E_{0}-\hbar \omega_{\sigma}\right)\left(E_{n}-E_{0}-\hbar \omega_{2}\right)}\right\}
\end{aligned}
$$

where $i, j$, and $k$ are Cartesian coordinate indices. The operator $\tilde{j}=j-\langle 0|j| 0\rangle, E_{m}$ is the energy of excited state $m$, $E_{0}$ is the energy of the ground state (0), $E_{m}-E_{0}$ is the transition energy between the ground state and excited state $m,\langle 0|i| m\rangle$ is the transition dipole between the ground state and excited state $m,\langle m|i| n\rangle$ is the transition dipole between excited states $m$ and $n, \omega_{1}$ and $\omega_{2}$ are incident photon frequencies, and $\omega_{\sigma}$ is the output photon frequency (e.g., $\omega_{\sigma}$ $=\omega_{1}+\omega_{2}$ for second harmonic generation). $P$ permutes the pairs $\left\{i,-\omega_{\sigma}\right\},\left\{j, \omega_{1}\right\},\left\{k, \omega_{2}\right\}$. The sum over permutations produces six terms.

Ratner et al. segregated the two-level and three-level contributions to $\beta_{\lambda},{ }^{52,53}$

$$
\beta_{i j k}=\sum_{n=1} \beta_{n}+\sum_{n=1} \sum_{m \neq n} \beta_{n m}
$$

where $\beta_{n}$ and $\beta_{n m}$ represent, respectively, state-specific two-level and three-level contributions to $\beta_{i j k}$. $\beta_{n}$ is the two-level contribution, arising from a sequence of transitions $(0 \rightarrow n \rightarrow n \rightarrow 0)$ between the ground state and excited state $n$ (Figure 1). Note that the two-state approximation (eq 4) is a special case of the SOS expression where only a single two-level term from the first summation dominates, and the photon energy is small compared to electronic transition energies. $\beta_{n}$ contains the transition dipole between the ground and excited states $\left(x_{0 n}\right.$ or $x_{n 0}$ ), as well as the dipole moment difference $\bar{x}_{n n}=x_{n n}-x_{00}$, where $x_{n n}$ is the dipole moment of excited state $n$, and $x_{00}$ is the dipole moment of ground state (0). In contrast, the three-level contribution $\beta_{n m}(n \neq m)$ arises from a sequence of transitions 0 $\rightarrow n \rightarrow m \rightarrow 0$. Evaluation of $\beta_{n m}$ requires transition dipoles between the ground and excited states $\left(x_{0 n}\right.$ and $\left.x_{m 0}\right)$ as well as transition dipoles among excited states $n$ and $m\left(x_{n m}\right)$. Excitedto-excited state transition dipoles are absent in the two-level terms.
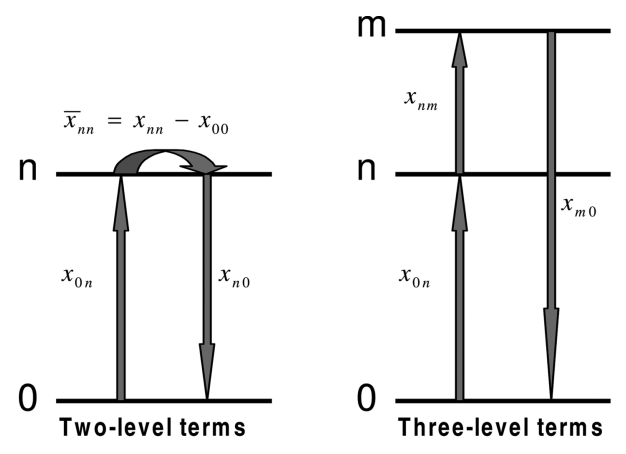

(a) $0 \rightarrow n \rightarrow n \rightarrow 0$ transitions. (b) $0 \rightarrow n \rightarrow m \rightarrow 0$ transitions.

Figure 1. Transition diagrams for (a) two-level terms $(0 \rightarrow n \rightarrow n \rightarrow$ 0 ) or intraband transitions and (b) three-level terms $(0 \rightarrow n \rightarrow m \rightarrow 0)$ or interband transitions in the SOS expression. The index 0 represents the ground electronic state, and indices $n$ and $m$ represent the excited electronic states. The electric dipole integral between any two states is $x_{p q}=\langle p|\hat{x}| q\rangle$. The dipole moment difference is $\bar{x}_{n n}=x_{n n}-x_{00}$, where $x_{n n}$ is the dipole moment of excited state $n$ and $x_{00}$ is the dipole moment of ground state 0 .

The two-level approximation for $\beta$ (retaining the first summation in eq 2) is ${ }^{19,24}$

$$
\beta \approx \sum_{n} \beta_{n}=\sum_{n} \frac{6\left(x_{0 n}\right)^{2} \bar{x}_{n n}\left(E_{0 n}\right)^{2}}{\left[\left(E_{0 n}\right)^{2}-\left(2 E_{i n c}\right)^{2}\right]\left[\left(E_{0 n}\right)^{2}-E_{\mathrm{inc}}^{2}\right]}
$$

where $E_{0 n}=E_{n}-E_{0}$ is the transition energy and $E_{\text {inc }}=\hbar \omega_{1}=$ $\hbar \omega_{2}$ is the energy of incident photons. By use of this two-level approximation with just two excited states, state-specific contributions to $\beta_{\lambda}$ for the archetypal D-PZn-A chromophore were explained qualitatively. ${ }^{19,21,23}$ Here, we will compute $\beta_{\lambda}$ by explicitly including two-level and three-level terms. When only one excited state is included in eq 3 and the radiation frequency goes to zero, the two-level approximation (eq 3) simplifies to the popular two-state approximation ${ }^{54,55}$

$$
\beta \propto\left(x_{n n}-x_{00}\right) \frac{x_{0 n}^{2}}{\left(E_{n}-E_{0}\right)^{2}}
$$

which is widely used for qualitative interpretations of NLO data. ${ }^{2,56,57}$ The two-state approximation is particularly valuable when $\beta$ is dominated by a small number of CT transitions that define a single electronic absorption manifold. For somewhat more complex chromophores, the two-state approximation fails to explain the frequency dispersion of $\beta$. This provides motivations for the present study.

Although Ratner et al. ${ }^{53,58}$ showed that three-level contributions to $\beta$ are not negligible, there are few direct experimental reports of excited state absorption spectra in relevant chromophores that could enable the direct experimental estimation of three-level contributions to $\beta$. Our strategy is to use the generalized Thomas-Kuhn sum (TKS) rules to extract unknown excited state-to-excited state transition dipole terms, $x_{n m}$, from experimental linear-absorption spectra and thereby estimate frequency dependent hyperpolarizabilities that include both twoand three-level contributions. While the number of effective excited states that we include will be small (typically two or three), both two and three-level contributions will be taken into account. 
The TKS rule that links the integrated oscillator strength to the total number of electrons is derived from diagonal commutator matrix elements of the molecular Hamiltonian with the position operator. ${ }^{59}$ The generalized TKS rules are corresponding off-diagonal matrix element commutator identities. These generalized TKS rules were used to great advantage in previous NLO studies by Kuzyk. ${ }^{13,14}$ These sum rules were used to obtain the fundamental limit of the hyperpolarizability and to test the validity of quantum calculations of excited states ${ }^{60-63}$ the offdiagonal sum rules were used to derive the "dipole-free" SOS expression ${ }^{14}$ and to relate the first and second hyperpolarizabilities to the linear electronic polarizability $(\alpha){ }^{64}$

The generalized TKS rules relate transition dipoles among excited states to transition energies and transition dipoles between the ground and excited states. Since transition dipoles and energies are readily computed from experimental linearabsorption spectra, the TKS rules can be used to predict frequency dependent hyperpolarizabilities, including both twoand three-level contributions. This TKS-SOS approach, employing linear absorption data, is particularly appealing because it circumvents the computation of electronic excited states. This is the first time the TKS-SOS approach has been used to interpret the three-level contributions (see eq 6) to $\beta_{\lambda}$ and thereby predict the full $\beta_{\lambda}$ spectrum by computing transition dipole moments among excited states using TKS rules. ${ }^{65}$

We now describe an approach to calculate transition dipoles among excited states using the generalized TKS rules and data in the linear absorption spectra. In addition to the experimental data, we employ two additional parameters, a transition energy shift factor $\left(S_{v}\right)$ and an absorption band half-width parameter $(\Gamma)$. These two factors adjust the extracted transition energies and transition dipoles because of offsets due to Franck-Condon envelopes and excited state lifetimes. By use of one measured $\beta_{\lambda}$ value (for the case of two dominant electronic absorption bands) or two measured $\beta_{\lambda}$ values (for the case of three dominant electronic transition manifolds), the entire $\beta_{\lambda}$ spectrum can be predicted. In cases where sufficient experimental data are available, the predicted $\beta_{\lambda}$ values are found to be consistent with the measured $\beta_{\lambda}$ values. This approach is applied to the $\beta_{\lambda}$ spectra of [5-[[4'-(dimethylamino)phenyl $]$ ethynyl $]-15-\left[\left(4^{\prime \prime}-\right.\right.$-nitrophenyl)ethynyl]-10,20-diphenylporphinato]zinc(II) (D-PZnA), ${ }^{21}$ 5-(4'-ethynyl-(2,2'; $6^{\prime}, 2^{\prime \prime}$-terpyridinyl $\left.)\right)-10,20$-bis $\left(2^{\prime}, 6^{\prime}\right.$ bis(3,3-dimethyl-1-butyloxy)phenyl) porphinato] zinc(II)$\left(2,2^{\prime} ; 6^{\prime}, 2^{\prime \prime} \text {-terpyridine }\right)^{2+}$ bis-hexafluorophosphate (Ru-PZn), and 5 -( $4^{\prime}$-ethynyl- $\left(2,2^{\prime} ; 6^{\prime}, 2^{\prime \prime}\right.$-terpyridinyl $\left.)\right)-10,20$-bis- $\left(2^{\prime}, 6^{\prime}\right.$ bis(3,3-dimethyl-1-butyloxy)phenyl)porphinato]zinc(II)$\left(2,2^{\prime} ; 6^{\prime}, 2^{\prime \prime} \text { - terpyridine }\right)^{2+}$ bis-hexafluoro-phosphate (Os-PZn). ${ }^{24}$ In this approach, both two-level and three-level contributions to $\beta_{\lambda}$ are taken into account and clarify the frequency dispersion of $\beta_{\lambda}$. The approximations associated with this approach and its limitations are also examined.

\section{Theory and Methods}

Two-Level and Three-Level Contributions to $\boldsymbol{\beta}$. The $\beta$ tensor component $\left(\beta_{x x x}\right)$ in the donor-acceptor $(\mathrm{D}-\mathrm{A})$ direction ( $x$-direction) often dominates the NLO response in large- $\beta$ molecules with donor-bridge-acceptor (DBA) motifs, and this is the case for the structures examined here. ${ }^{21}$ We therefore use $\beta_{x x x}$ to evaluate the molecular hyperpolarizabilities. The groundto-excited state transition energies and dipole moments along the $x$ direction, required for $\beta_{x x x}$ calculations, are extracted from measured linear absorption manifolds. Then, the transition dipole moments among excited states in the $\mathrm{x}$-direction can be computed using the generalized TKS rules.
In eq 2 , the two-level $\beta$-contributions are

$$
\beta_{n}=-x_{0 n} \bar{x}_{n n} x_{n 0} D_{n n}\left(\omega_{1}, \omega_{2}\right)
$$

and the three-level $\beta$-contributions are

$$
\beta_{n m}=-x_{0 m} x_{m n} x_{n 0} D_{m n}\left(\omega_{1}, \omega_{2}\right)
$$

where $D_{m n}\left(\omega_{1}, \omega_{2}\right)$ is a function of the incident frequencies $\omega_{1}$ and $\omega_{2} \cdot{ }^{66}$ Specifically

$$
D_{m n}=\frac{1}{2 \hbar^{2}}\left[d_{m n}\left(\omega_{1}, \omega_{2}\right)+d_{m n}\left(\omega_{2}, \omega_{1}\right)\right]
$$

where

$$
\begin{gathered}
d_{m n}\left(\omega_{1}, \omega_{2}\right)=\frac{1}{\left(\omega_{m 0}-\omega_{1}-\omega_{2}\right)\left(\omega_{n 0}-\omega_{1}\right)}+ \\
\frac{1}{\left(\omega_{m 0}^{*}+\omega_{2}\right)\left(\omega_{n 0}-\omega_{1}\right)}+ \\
\frac{1}{\left(\omega_{m 0}^{*}+\omega_{2}\right)\left(\omega_{n 0}^{*}+\omega_{1}+\omega_{2}\right)}
\end{gathered}
$$

Lifetime terms $\left(\gamma_{m 0}\right)$ are added to the transition energies $\left(\omega_{m 0}\right)$ : $\omega_{m 0}=\omega_{m 0}^{0}-i \gamma_{m 0}$, where $\hbar \omega_{m 0}^{0} \equiv E_{0 m}=E_{m}-E_{0}$.

Transition Energies and Transition Dipoles from Linear Absorption Spectra. Transition dipoles (in Debyes) and energies (in wavenumbers) are related to oscillator strengths by ${ }^{67}$

$$
f_{0 n}=4.702 \times 10^{-7} \tilde{E}_{0 n}\left|x_{0 n}\right|^{2}=6.784 \times 10^{-9} \varepsilon_{\max }^{0 n} \Gamma
$$

where $f_{0 n}$ is the oscillator strength for the ground to excited state $n$ transition, $E_{0 n}$ is the transition energy in wavenumbers, and $\varepsilon_{\max }^{0 n}$ is the extinction coefficient in $\mathrm{dm}^{3} \mathrm{~mol}^{-1} \mathrm{~cm}^{-1}$ at the absorption maximum. $\Gamma$ is the width of the absorption band at $\varepsilon_{\max }^{0 n} / 2$, and a Gaussian line shape is assumed. $\left|x_{0 n}\right|$ can be computed from measured spectra using eq 8. $x_{0 n}$ (eq 8) is taken positive (the input signs of transition dipoles do not affect our results; see Table S5 of Supporting Information). Experimentally measured absorption bands may peak at energies above the electronic origin because of the Franck-Condon envelope. ${ }^{67}$ As such, we adjust $E_{0 n}$ by a shift factor $S_{v}\left(S_{v}<1\right)$

$$
E_{n}=S_{v} E_{0 n}
$$

The transition dipoles and transition energies provide two of the key ingredients for computing $\beta$. The missing ingredients in the SOS expression are the transition elements among excited states, which will now be computed using the generalized TKS rules.

Generalized TKS Rules and Their Truncated Expressions. The generalized TKS rule ${ }^{13,14}$ is

$$
\sum_{n=0}^{\infty}\left(2 E_{n}-E_{k}-E_{l}\right) x_{k n} x_{n l}=\frac{\hbar^{2} N}{m_{\mathrm{e}}} \delta_{k l}
$$

where $k, l$, and $n$ are electronic states indices, $\hbar$ is the Planck's constant divided by $2 \pi, N$ is the total number of electrons, $m_{\mathrm{e}}$ 
is the electron mass, and $\delta$ is the Kronecker delta function. When $k=l=0$, the standard TKS rule results; when $k \neq l$, the generalized sum rule obtains. The generalized sum rule (eq 10) truncated to a finite number $(M)$ of excited states is

$$
\sum_{n=0}^{M}\left(2 E_{n}-E_{k}-E_{l}\right) x_{k n} x_{n l}=\frac{\hbar^{2} N}{m_{\mathrm{e}}} \delta_{k l}
$$

The truncated diagonal sum rule $(k=l)$

$$
2 \sum_{n=0}^{M}\left(E_{n}-E_{k}\right) x_{n k}^{2}=\frac{\hbar^{2} N}{m_{\mathrm{e}}}
$$

contains a paradox. ${ }^{68}$ If $k=M$, the left side of eq 12 is negative because $E_{n}-E_{M} \leq 0$, but the right side is positive because all the physical constants are positive. This inconsistency arises from truncation and is avoided if $k$ is the ground state, since all excited-state energies are larger than the ground state energy.

The off-diagonal sum rule $(k \neq l)$

$$
\sum_{n=0}^{M}\left(2 E_{n}-E_{k}-E_{l}\right) x_{k n} x_{n l}=0
$$

links the transition dipoles between excited states $m$ and $n$ to transition dipoles between the ground state and excited states and to the corresponding excitation energies. The latter two terms can be computed or derived from the linear-absorption spectra, as described above.

In quantum calculations, Champagne and Kirtman found that the truncated diagonal sum rules may not be obeyed. ${ }^{69}$ Kuzyk later explained that this discrepancy was caused by errors in the computed excited-state wave functions. ${ }^{61}$ The truncated offdiagonal sum rules have been shown to be approximately valid $^{61,69}$ and were used to successfully calculate $\beta$ based on dipole-free SOS formulas. ${ }^{13,14,65,70}$ As shown in the discussion of truncation errors in section 3 of the Supporting Information, transition dipole moments among excited states are just linearly scaled with respect to the truncation errors of off-diagonal sum rules, as are the predicted hyperpolarizabilities. The relative ratios of the predicted dipole moments and the trends in the predicted frequency-dependent $\beta$-dispersions are not critically influenced by the truncation error. Moreover, the neglect of highenergy excited states in our TKS-SOS method has little influence on the predicted transition dipoles and the dynamic $\beta$ spectra.

For molecules near the fundamental limit of the static hyperpolarizability, $\beta$ can be calculated using a three level ansatz $^{60,61}$ (i.e., a ground state and two excited states). In the porphyrin-based NLO chromophores studied here, the experimental absorption spectra are consistent with the three-level ansatz, as two major excited state manifolds are found. Specifically, D-PZn-A manifests a value of $\beta_{0} / N^{3 / 2}\left(\beta_{0} / N^{3 / 2} \approx 30 \times\right.$ $10^{-30} \mathrm{esu}$, where $N$ is the number of effective electrons) beyond the "apparent limit" and close to the "fundamental limit," constraints on the hyperpolarizabilities noted in recent studies of Kuzyk and co-workers based on sum rule analysis. ${ }^{71}$ In addition, Figure S2 of Supporting Information indicates that truncating to the top three excited states with the strongest oscillator strength (computed using TD-DFT/B3LYP methods with the collective electronic oscillator ${ }^{40}$ description for D-PZn-
A) is sufficient to predict the entire $\beta_{\lambda}$ spectrum. Thus, the offdiagonal sum rules for $\mathbf{D}-\mathbf{P Z n}-\mathbf{A}$ are truncated to include only the ground state and two excited states (i.e., the three-level ansatz). For the Ru-PZn and Os-PZn molecules, which feature $\beta_{0} / N^{3 / 2}$ values $\left(\beta_{0} / N^{3 / 2} \approx 7 \times 10^{-30}\right.$ esu $)$ beyond the apparent limit, ${ }^{71}$ the off-diagonal sum rules are truncated to four levels including the ground state and three excited states to predict the $\beta_{\lambda}$ spectrum.

Possible Solutions based on TKS Rules. The truncated offdiagonal sum rule $(k \neq l)$ of eq 11 produces $M(M+1) / 2$ quadratic equations that link transition-dipole elements to each other and to transition energies $(k=0,1, \ldots, M ; l=0$, ..., $k-1)$

$$
\left\{\begin{array}{c}
\sum_{n=0}^{M}\left(2 E_{n}-E_{1}-E_{0}\right) x_{1 n} x_{n 0}=0, \quad k=1, l=0 \\
\sum_{n=0}^{M}\left(2 E_{n}-E_{2}-E_{0}\right) x_{2 n} x_{n 0}=0, \quad k=2, l=0 \\
\sum_{n=0}^{M}\left(2 E_{n}-E_{2}-E_{1}\right) x_{2 n} x_{n 1}=0, \quad k=2, l=1 \\
\vdots \\
\sum_{n=0}^{M}\left(2 E_{n}-E_{2}-E_{0}\right) x_{(M) n} x_{n(M-1)}=0 \\
k=M, l=M-1
\end{array}\right.
$$

The number of transition dipole elements $\left(x_{m n}, m, n \neq 0\right)$ in these equations that can not be determined by the linearabsorption spectrum is $M(M+1) / 2$. The quadratic relation among transition dipoles produces multiple solutions for these elements. The maximum number of solutions for these elements, excluding the sign difference, is $2^{M(M-1) / 2-1}$. For spectra with two absorption bands $(M=2)$, there is one unique solution. For spectra with three key absorption bands, as Ru-PZn or Os-PZn, there are four possible solutions for the $x_{m n}$ elements. To simplify the programming complexity, only real $x_{m n}$ elements are considered in our model. For systems with more than one solution, we can use a few (e.g., one or two) experimental $\beta_{\lambda}$ values to determine the physically relevant solution and then quantitatively predict the full $\beta_{\lambda}$ spectrum.

The generalized sum rules provide a strategy to collect the missing $x_{m n}$ elements from the linear absorption data, thus allowing computation of $\beta_{\lambda}$ based entirely on experimental linear-absorption data and a truncated SOS expression for $\beta_{\lambda}$. The key assumptions underlying this approach are: (1) the features of the linear absorption spectra of dipolar chromophores arise from transitions predominantly polarized along the CT axis, and (2) electronic transitions in the UV/vis/NIR window dominate the SOS expressions and, as such, truncating the summations is acceptable.

\section{Results and Discussion}

1. Extracting Transition Energies and Transition Dipoles from Linear Absorption Spectra. Electronic absorption bands for large organic molecules in solution usually contain more than one electronic or vibronic transition. For example, in D-PZn-A, Stark spectroscopy ${ }^{19}$ indicates that two intense peaks contain seven electronic and vibronic excited states. Yet, it is impossible to identify all electronic and vibronic transitions from conventional experimental linear-absorption spectra. Thus, the analysis of dressed or effective transitions 
is useful. For instance, from one single measured band, Kuzyk and Clays ${ }^{15}$ extracted the dressed or effective transition dipole moments that were then used to determine contributions to $\beta_{0}$.

Here, we apply a similar effective transition strategy to extract information about each absorption band. In our strategy, the transition energies and dipoles are computed from the maximal absorption coefficient for each band using eqs 8 and 9. Even though each band includes extensive mixing of different electronic and vibronic transitions, for the compounds under study, the prominent absorption features have dominant contributions from specific electronic transitions. For example, the intense, lowest energy band in the D-PZn-A electronic spectrum is dominated by $x$-polarized $Q$-state transitions; likewise, the broad absorption band centered at $\sim 450 \mathrm{~nm}$ features high oscillator strength contributions dominated by $B_{x}$ transitions because the arylethynyl-based D and A moieties mix strongly with the PZn frontier orbitals, augmenting the $x$-polarized transition moments. ${ }^{19-21,23}$

The protocol to obtain the two parameters in our model is to scan the shift factor $S_{v}$ (eq 9) (between 0.80 and 1.00) and the absorption band half-width parameter $\Gamma$ (between 0.04 and $0.1 \mathrm{eV}$ ) and to solve the TKS equations for each set of $S_{v}$ and $\Gamma$ to calculate the $\beta_{\lambda}$ spectrum. $\left(S_{v}\right.$ was varied in step sizes of 0.01 and $\Gamma$ was varied in steps of $0.001 \mathrm{eV}$.) The finial values of the two parameters were chosen to best reproduce one measured $\beta_{\lambda}$ value (for the case of two linear absorption bands) or two measured $\beta_{\lambda}$ values (for the case of three linear absorption bands). Three experimental $\beta_{\lambda}$ values are available at 800,1064 , and $1300 \mathrm{~nm}$ for each NLO chromophore; the measured $\beta_{\lambda}$ value at $800 \mathrm{~nm}\left(\beta_{800}\right)$ for D-PZn-A was used to determine $S_{v}$ and $\Gamma$, while the measured $\beta_{\lambda}$ values at $800 \mathrm{~nm}$ and $1300 \mathrm{~nm}$ were used to compute the Ru-PZn and Os-PZn frequency-dependent hyperpolarizabilities. (For D-PZn-A, it is unimportant which $\beta_{\lambda}$ value is chosen to calibrate the $\beta_{\lambda}$ spectrum. For Ru-PZn and OsPZn, $\beta_{1300}$ must be chosen to distinguish the similar TKS solutions since the $\beta_{1300}$ value is drastically changed from Ru-PZn to Os-PZn. $\beta_{800}$ is also chosen because it is close to zero in the experimental measurements. $\beta_{1064}$ is predicted and compared to the experimental values.) The predicted $\beta_{\lambda}$ spectra were compared to the molecular hyperpolarizability measurements at the other experimentally studied wavelength. The damping constant was taken to be $0.1 \mathrm{eV}$, a typical choice for an excited state lifetime of $4.1 \mathrm{ps}^{72}$

2. Applying TKS Rules to an NLO Chromophore with Two Absorption Manifolds with CT Character. $\beta_{\lambda}$ for D-PZn-A was examined in earlier semiempirical studies, ${ }^{23}$ and state-specific $\beta_{\lambda}$ contributions were examined for the seven electronic and vibronic states using the two-level approximation. ${ }^{19}$ It is necessary to describe $\beta_{\lambda}$ with both twolevel and three-level contributions (see Figure 1) to understand the interaction between $x$-polarized $B$ and $Q$ states. The molecular structure, along with the D-PZn-A experimental linear-absorption spectrum, appears in Figure 2. The spectrum has just two key absorption manifolds, which are comprised of seven prominent electronic and vibronic transitions: ${ }^{19}$ $Q_{x}(0,0), Q_{y}(0,0), Q_{x}(1,0), B_{x}(0,0), B_{x}(1,0), B_{y}(0,0)$, and $B_{y}(1,0)$. As described above, we use two effective electronic transitions to predict the molecular hyperpolarizability.

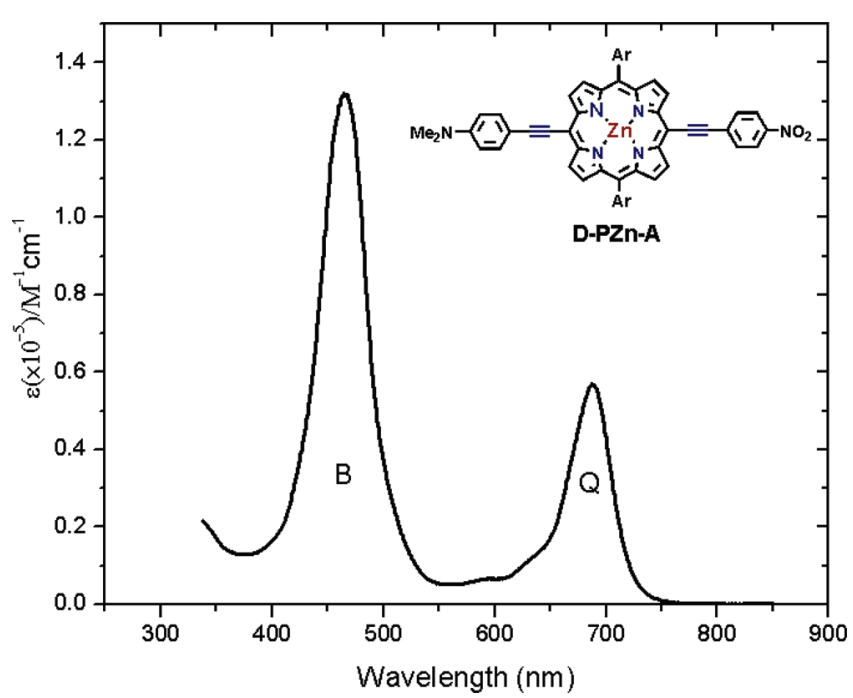

Figure 2. Molecular structure and experimental absorption spectrum of D-PZn-A in $\mathrm{CHCl}_{3}$ solvent. Reproduced with permission of ACS from ref 21 .

TABLE 1: Transition Energies ( $E_{01}$ and $E_{02}$ in $\left.\mathrm{eV}\right)$ and Transition Dipole Moments ( $x_{01}$ and $x_{02}$ in au) Obtained from the Linear Absorption Spectrum of D-PZn-A in $\mathrm{CHCl}_{3}$ Solvent $^{a}$

\begin{tabular}{cccccccc}
\hline & $E_{01}$ & $E_{02}$ & $x_{01}$ & $x_{02}$ & $\bar{x}_{11}$ & $\bar{x}_{22}$ & $x_{12}$ \\
\hline D-PZn-A & 1.68 & 2.48 & 2.18 & 2.74 & 9.27 & 1.06 & -3.78
\end{tabular}

${ }^{a}$ The transition moments ( $x_{12}$ in au) and dipole moment differences $\left(\bar{x}_{11}\right.$ and $\bar{x}_{22}$ in au) are computed by solving the generalized TKS equations for D-PZn-A with $S_{v}=0.93$ and $\Gamma=$ $0.063 \mathrm{eV}$ (fit to reproduce experimental $\beta_{800}$ ) and to predict $\beta_{1064}$ and $\beta_{1300}$.

TKS Rules for NLO Chromophores with Two Absorption Manifolds that Feature CT Character. The TKS rules (eq 14) for the two-bands produce the equations

$$
\left\{\begin{array}{l}
\left(E_{1}-E_{0}\right) \bar{x}_{11} x_{10}+\left(2 E_{2}-E_{1}-E_{0}\right) x_{12} x_{20}=0 \\
\left(2 E_{1}-E_{2}-E_{0}\right) x_{21} x_{10}+\left(E_{2}-E_{0}\right) \bar{x}_{22} x_{20}=0 \\
\left(2 E_{0}-E_{2}-E_{1}\right) x_{20} x_{01}+\left(E_{2}-E_{1}\right)\left(\bar{x}_{22}-\bar{x}_{11}\right) x_{21}=0
\end{array}\right.
$$

One unique solution of eq 15 for $\bar{x}_{11}, \bar{x}_{22}$, and $x_{12}\left(x_{21}\right)$ appears in Table 1. $\bar{x}_{11}$ and $\bar{x}_{22}$, which are dipole moment differences for the effective $Q$ and $B$ states, respectively, relative to the ground state have the same sign, since both the $x$-polarized porphyrin $Q$ and $B$ states possess similarly polarized CT character.

Predicted $\boldsymbol{\beta}_{\lambda}$ Spectrum of D-PZn-A. The predicted hyperpolarizability spectrum (Figure 3 ) agrees with experimental $\beta_{\lambda}$ values measured at 800,1064 , and $1300 \mathrm{~nm}$. The value at 800 $\mathrm{nm}$ was fit by scanning the parameters $S_{v}$ (from 0.8 to 1.0 ) and $\Gamma$ (from 0.04 to $0.1 \mathrm{eV}$ ). Table 2 shows the predicted values of $\beta_{\lambda}$ at 1064 and $1300 \mathrm{~nm}$, which agree well with the measured data (uncertainties in measured data are typically $\pm 10 \%$ ).

Influence of Two- and Three-Level Contributions to the Computed $\boldsymbol{\beta}_{\lambda}$. The computed frequency-dependent two- and three-level contributions to $\beta_{\lambda}$ are shown for D-PZn-A in Figure 3. $\beta_{1}$ is the two-level contribution from $Q_{x}$-derived transitions; $\beta_{2}$ is the two-level contribution from $B_{x}$-derived transitions; $\beta_{12}$ and $\beta_{21}$ are the three-level contributions from the coupling of $Q_{x^{-}}$and $B_{x}$-derived excited states. 


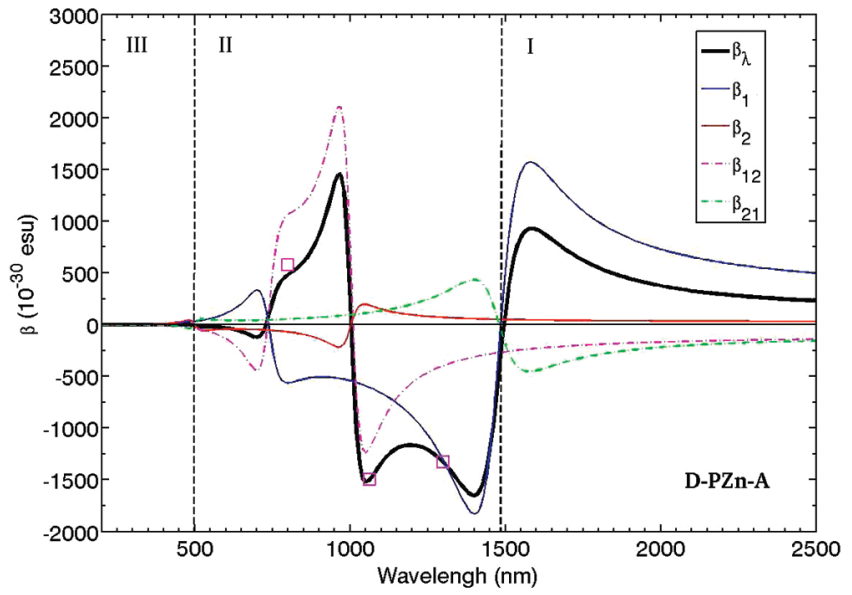

Figure 3. Computed $\beta_{\lambda}$ and its components $\beta_{n}$ and $\beta_{n m}$ from eqs 5 and 6 of D-PZn-A with shift factor $S_{v}=0.93$ and half-width parameter $\Gamma=0.063 \mathrm{eV}$. The open squares are measured $\beta_{\lambda}$ values at 800,1064 , and $1300 \mathrm{~nm}$ in $\mathrm{CH}_{2} \mathrm{Cl}_{2}$ solvent. ${ }^{73}$ The irradiation wavelength ranges are divided into three regions (I, II, and III).

TABLE 2: Calculated and Measured $\beta_{\lambda}\left(10^{-30} \mathrm{esu}\right)$ for D-PZn-A in $\mathrm{CH}_{2} \mathrm{Cl}_{2}$ Solvent $^{73}$

\begin{tabular}{cccc}
\hline D-PZn-A & $\beta_{800}$ & $\beta_{1064}$ & $\beta_{1300}$ \\
\hline calcd & 479 & 1489 & 1326 \\
exptl & 580 & 1500 & 1330
\end{tabular}

Each contribution that enters $\beta_{\lambda}$ has one-photon and twophoton resonant enhancements, described by the $D_{m n}$ functions in eq 7. $D_{m n}$ changes sign at the two resonance frequencies (see Figure 4). Two-photon resonance arises at $\left(\omega_{m 0}-\omega_{1}-\omega_{2}\right)=$ 0 and one-photon resonance arises at $\left(\omega_{n 0}-\omega_{1}\right)=0$. One positive and one negative peak appear on each side of the resonance frequency.

Although $D_{m n}$ determines the frequency dependence of $\beta_{n}$ and $\beta_{n m}$, the magnitude of $\beta_{n}$ and $\beta_{n m}$ is controlled by the two-level terms $x_{0 n} \bar{x}_{n n} x_{n 0}(n=m)$ and the three-level terms $x_{0 n} \bar{x}_{n m} x_{m 0}(n \neq$ $m$ ). Prior studies have used the two-level approximation ${ }^{19,21,23}$ to evaluate the molecular hyperpolarizability. In the following analysis, we show that the three-level contributions can be essential for describing the frequency dependent hyperpolarizability.

Near $800 \mathrm{~nm}$ (Figure 3II), the three-level terms, $\beta_{12}$ (from the $0 \rightarrow Q_{x} \rightarrow B_{x} \rightarrow 0$ transition) and $\beta_{21}$ (from the $0 \rightarrow B_{x} \rightarrow$ $Q_{x} \rightarrow 0$ transition), eq 6 , both have opposite signs from the

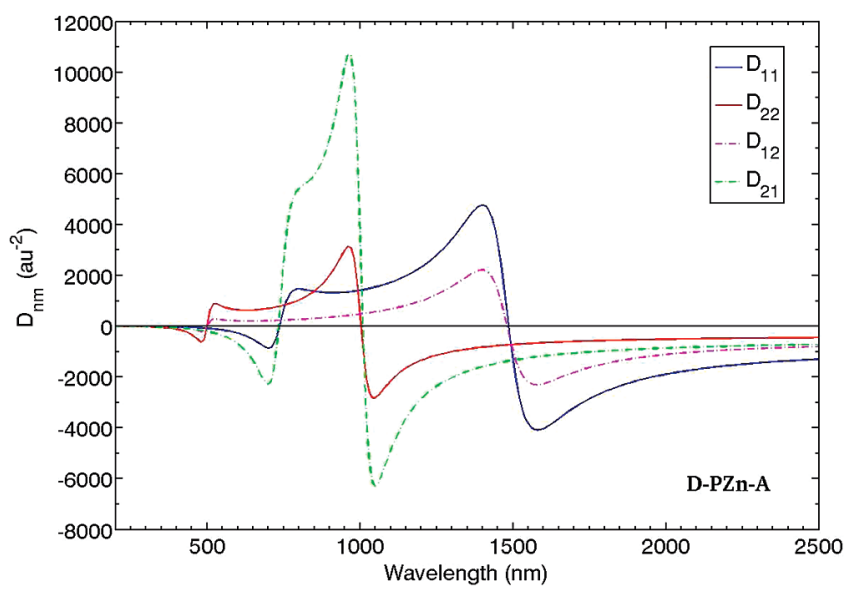

Figure 4. Wavelength dependence of $D_{m n}$ (atomic units, eq 7) for D-PZn-A.

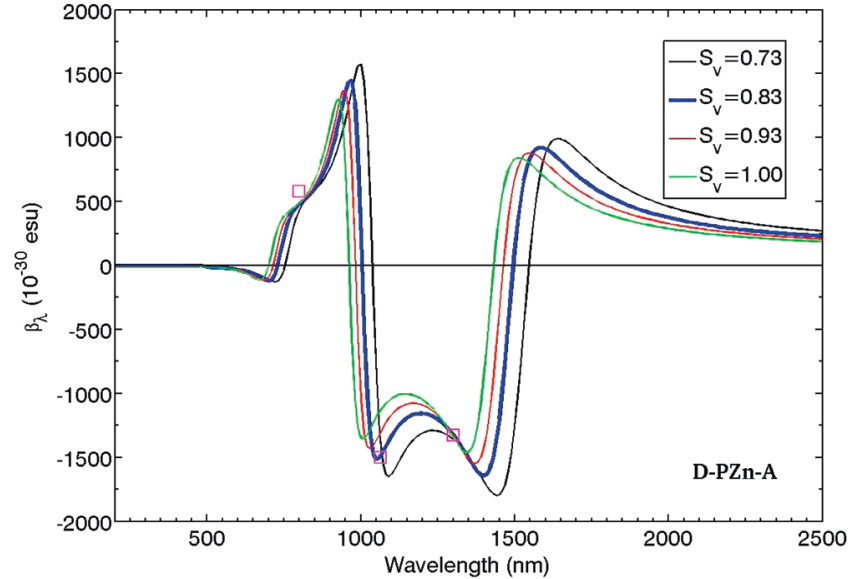

Figure 5. $\beta_{\lambda}$ spectra of D-PZn-A for a range of $S_{v}$ factors. $\Gamma=0.063 \mathrm{eV}$.

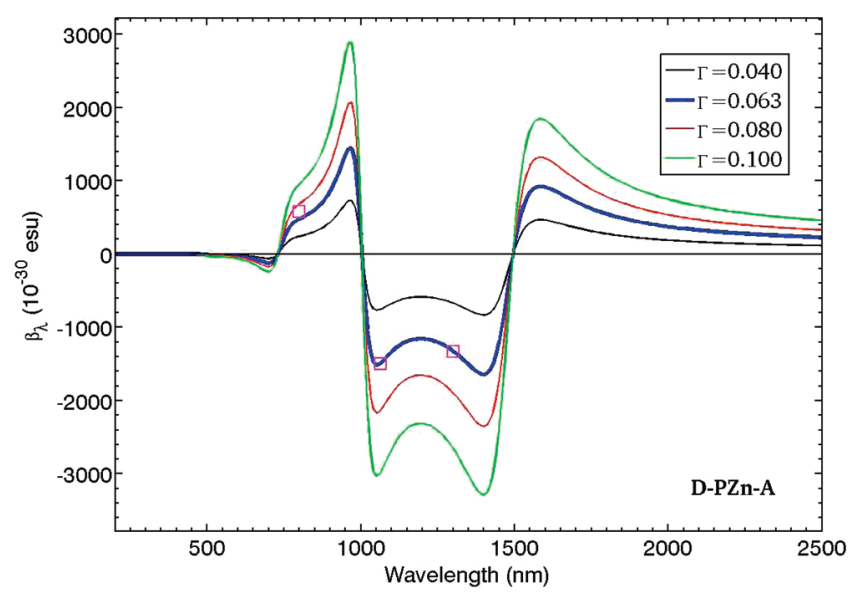

Figure 6. $\beta_{\lambda}$ spectra of D-PZn-A computed for representative halfwidth parameters. $S_{v}=0.93$.

two-level terms, $\beta_{1}$ (from the $0 \rightarrow Q_{x} \rightarrow Q_{x} \rightarrow 0$ transition), and $\beta_{2}$ (from the $0 \rightarrow B_{x} \rightarrow B_{x} \rightarrow 0$ transition), eq 5 . Because the two- and three-level contributions nearly cancel at $800 \mathrm{~nm}$, $\beta_{800}$ is very small. At $1064 \mathrm{~nm}$, the three-level contribution $\beta_{12}$ dominates, so the excited state transition dipole moment $\left(x_{12}\right)$ enhances $\beta$. At $1300 \mathrm{~nm}, \beta$ is dominated by the $Q_{x}$-state contribution because of the large dipole moment difference $\left(\bar{x}_{11}\right.$ $=9.27$ ) between the $Q_{x}$-state and the ground state. Thus, $\beta_{1300}$ is controlled mainly by the two-level contribution, $\beta_{1}$, derived from the $Q_{x}$-state. Indeed, hyper-Raleigh light scattering experiments indicate that the measured response at $1300 \mathrm{~nm}$ is dominated by $Q_{x}$-state contributions for a series of (porphinato)zinc(II)-based donor-acceptor chromophores. ${ }^{26,27}$

For D-PZn-A, where the absorption spectrum is dominated by two manifolds that possess charge-resonance character, we find that the three-level $\beta_{n m}$ contributions $(n \neq m)$ cancel twolevel contributions and diminish the magnitude of $\beta_{800}$, but enhance $\beta$ at $1064 \mathrm{~nm}$.

Sensitivity of $\beta_{\lambda}$ to the Magnitudes of $S_{v}$ and $\Gamma$. Figures 5 and 6 show that the qualitative $\lambda$ dependence of $\beta$ is unchanged when the magnitudes of $S_{v}$ and $\Gamma$ are varied. The spectral shift factor red shifts the computed $\beta_{\lambda}$ spectrum with increasing $S_{v}$ values (Figure 5). Increasing the half-width parameter, which increases the transition dipole moments, increases $\left|\beta_{\lambda}\right|$ (Figure 6).

For $S_{v}$ and $\Gamma$ within the range shown in Figures 5 and $6(0.7$ $\leq S_{v} \leq 1.0$ and $0.04 \leq \Gamma \leq 1.0$ for D-PZn-A), the predicted $\beta_{\lambda}$ spectra are weakly $S_{v}$ and $\Gamma$ dependent. The exact $\beta_{\lambda}$ values at 


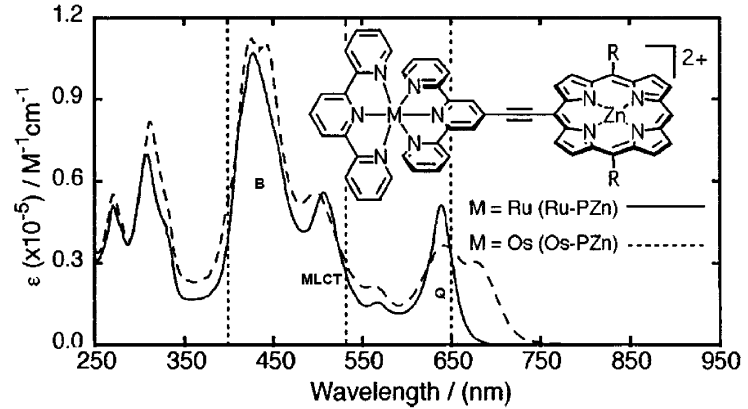

Figure 7. Molecular structures and linear absorption spectra of Ru-PZn and Os-PZn in $\mathrm{CH}_{3} \mathrm{CN}$ solvent. Reproduced with permission of ACS from ref 24.

different wavelengths depend on both $S_{v}$ and $\Gamma$. In summary, the exact positions of the electronic one- or two-photon resonances in the $\beta_{\lambda}$ spectra are controlled by $S_{v}$. The contributions to the $\beta_{\lambda}$ values that are derived from the measured linear absorption manifolds are adjusted by $\Gamma$.

3. Applying TKS Rules to an NLO Chromophore with Three Absorption Manifolds with Charge-Resonance Character. M-PZn chromophores feature (porphinato)zinc(II) (PZn) and metal(II)terpyridyl (M) units linked via an ethyne unit that bridges the meso-macrocycle and 4'-terpyridyl positions. This connectivity aligns the high oscillator strength transition dipoles in a head-to-tail motif, creating a supermolecule with coupledoscillator photophysics. ${ }^{9,17,24,28,74}$ Previous studies established that this motif enables elaboration of NLO chromophores with exceptionally large hyperpolarizabilities at telecommunication relevant wavelengths. ${ }^{17,24}$ In addition, it was recognized that these structures can be designed so that $B$ - and $Q$-state-derived $\beta_{\lambda}$ contributions can have the same or opposite sign; this effect, coupled with the fact that the sign of the resonance enhancement factor is frequency dependent, gives rise to NLO chromophores with unusual oscillatory frequency dependent hyperpolarizabilities. ${ }^{24} \mathbf{R u}-\mathbf{P Z n}$ and $\mathbf{O s - P Z n}$ are a particularly interesting pair because they possess similar linear-absorption spectra but have dramatically different dynamic hyperpolarizabilities. ${ }^{24}$ As such, these systems pose a challenging test for this theoretical approach.

The molecular structures and linear-absorption spectra of RuPZn and Os-PZn are shown in Figure 7. The electronic spectra evince strong mixing of PZn-based oscillator strength with metal polypyridyl charge-resonance bands and display a variety of new low-energy electronic transitions that feature significant transition dipole strengths and large extinction coefficients. This strong mixing gives rise to three absorption manifolds originating in (porphinato)zinc $B$ - and $Q$-states as well as in (terpyridyl)metal metal-to-ligand charge transfer (MLCT) states, while manifesting substantial interpigment charge-resonance interactions in the supermolecule.

We show that the NLO response of PZn- and (terpyridyl) metal-derived species can be described quantitatively using the computed three-level contributions to compute the entire $\beta_{\lambda}$ spectra of Ru-PZn and Os-PZn. This generalized sum rule analysis, therefore, describes the physical origins of the unusual hyperpolarizability frequency dispersion exhibited by these highly polarizable chromophores.

TKS Rules for Species with Three Absorption Manifolds that Feature CT Character. The $B_{x}, Q_{x}$, and MLCT-derived absorption manifolds that feature charge-resonance interactions in the Ru-PZn and Os-PZn supermolecules can be treated
TABLE 3: Transition Energies $\left(E_{01}, E_{02}\right.$, and $E_{03}$ in $\left.\mathrm{eV}\right)$ and Transition Moments ( $x_{01}, x_{02}$, and $x_{03}$ in au) Obtained from the Linear Absorption Spectra Measured in $\mathrm{CH}_{3} \mathrm{CN}$ Solvent ${ }^{a}$

\begin{tabular}{lrr}
\hline & Ru-PZn $^{b}$ & Os-PZn $^{c}$ \\
\hline$E_{01}$ & 1.59 & 1.52 \\
$E_{02}$ & 2.01 & 2.07 \\
$E_{03}$ & 2.37 & 2.42 \\
$x_{01}$ & 2.66 & 1.62 \\
$x_{02}$ & 2.48 & 1.91 \\
$x_{03}$ & 3.16 & 2.49 \\
$\bar{x}_{11}$ & -12.58 & 7.25 \\
$\bar{x}_{22}$ & 17.05 & 4.26 \\
$\bar{x}_{33}$ & 5.53 & 2.41 \\
$x_{12}$ & 4.63 & 3.24 \\
$x_{13}$ & 2.54 & -4.12 \\
$x_{23}$ & 11.50 & -3.17
\end{tabular}

${ }^{a}$ Transition moments $\left(x_{12}, x_{13}\right.$, and $\left.x_{23}\right)$ among excited sates and dipole moment differences $\left(\bar{x}_{11}, \bar{x}_{22}\right.$, and $\bar{x}_{33}$ in au) are computed by the TKS equations for Ru-PZn and Os-PZn. $S_{v}$ is the shift factor and $\Gamma$ is the half-width parameter. $S_{v}$ and $\Gamma$ were fitted to reproduce experimental $\beta_{800}$ and $\beta_{1300}$ and predicted $\beta_{1064}$ and $\beta_{1500}$ values, respectively. ${ }^{b} S_{v}=0.82, \Gamma=0.099 \mathrm{eV} .{ }^{c} S_{v}=0.83, \Gamma=0.06 \mathrm{eV}$.

within the context of the generalized sum rules (eq 14), which link transition-dipoles, transition energies, and dipole moments.

$$
\left\{\begin{array}{c}
\left(E_{1}-E_{0}\right) \bar{x}_{11} x_{10}+\left(2 E_{2}-E_{1}-E_{0}\right) x_{12} x_{20}+ \\
\left(2 E_{3}-E_{1}-E_{0}\right) x_{13} x_{30}=0 \\
\left(2 E_{1}-E_{2}-E_{0}\right) x_{21} x_{10}+\left(E_{2}-E_{0}\right) \bar{x}_{22} x_{20}+ \\
\left(2 E_{3}-E_{2}-E_{0}\right) x_{23} x_{30}=0 \\
\left(2 E_{0}-E_{2}-E_{1}\right) x_{20} x_{01}+\left(E_{2}-E_{1}\right)\left(\bar{x}_{22}-\bar{x}_{11}\right) x_{21}+ \\
\left(2 E_{3}-E_{2}-E_{1}\right) x_{23} x_{31}=0 \\
\left(2 E_{1}-E_{3}-E_{0}\right) x_{31} x_{10}+\left(2 E_{2}-E_{3}-E_{0}\right) x_{32} x_{20}+ \\
\left(E_{3}-E_{0}\right) \bar{x}_{33} x_{30}=0 \\
\left(2 E_{0}-E_{3}-E_{1}\right) x_{30} x_{01}+\left(2 E_{2}-E_{3}-E_{1}\right) x_{32} x_{21}+ \\
\left(E_{3}-E_{1}\right)\left(\bar{x}_{33}-\bar{x}_{11}\right) x_{31}=0 \\
\left(2 E_{0}-E_{3}-E_{2}\right) x_{30} x_{02}+\left(2 E_{1}-E_{3}-E_{2}\right) x_{31} x_{12}+ \\
\left(E_{3}-E_{2}\right)\left(\bar{x}_{33}-\bar{x}_{22}\right) x_{32}=0
\end{array}\right.
$$

All of the associated excited state transition dipoles were computed using eq 16 and the experimental absorption spectra. Four possible solutions were obtained, as three excited states are considered. By use of measured $\beta_{\lambda}$ values at 800 and 1300 $\mathrm{nm}$, the transition dipoles among excited states were computed (Table 3) for Ru-PZn and Os-PZn. We found that $B_{x^{-}}$and $Q_{x^{-}}$ state-derived dipole moment differences $\bar{x}_{n n}$ indeed have opposite signs (Table 3) for Ru-PZn: $\bar{x}_{33}$ is 5.53 au (atomic units) and $\bar{x}_{11}$ is $-12.58 \mathrm{au}$. In contrast, for Os-PZn, we found that the $B$ and $Q$-state $\bar{x}_{n n}$ values have the same sign: $\bar{x}_{33}=2.41$ au and $\bar{x}_{11}=7.25 \mathrm{au}$, which are smaller than those determined for Ru-PZn. In addition, the $\bar{x}_{n n}$ values for $B_{x^{-}}$and $Q_{x}$-derived supermolecular charge resonances are of opposite sign from the excited-to-excited transition dipoles $\left(x_{13}\right.$ and $\left.x_{23}\right)$ in Os-PZn. For $B_{x}$ - and $Q_{x}$-states, $x_{13}=-4.12 \mathrm{au}$; for the (terpyridyl)metal MCLT- and $Q_{x}$-derived supermolecular charge-resonance states, $x_{23}=-3.17 \mathrm{au}$. As we will see below, the sign changes evident in the calculated Os-PZn dipole moment differences will play an important role in determining the observed dynamic hyperpolarizability and the computed $\beta_{\lambda}$ spectrum.

Predicted $\boldsymbol{\beta}_{\lambda}$ Spectra of M-PZn Chromophores. Figures 8 and 9 show the predicted $\beta_{\lambda}$-spectra for $\mathbf{R u}-\mathbf{P Z n}$ and Os-PZn, together with the computed $\beta_{n m}$ for each chro- 


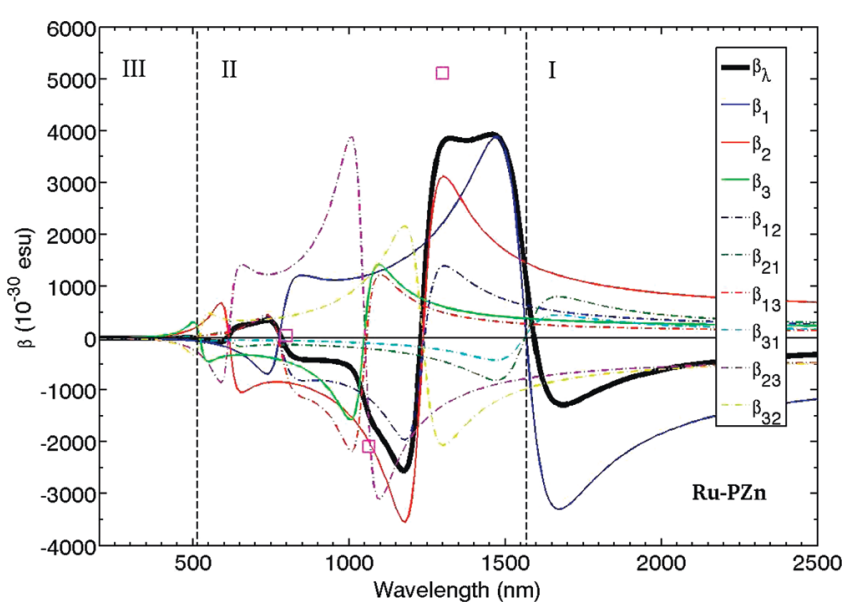

Figure 8. Computed $\beta_{\lambda}$ and its components $\beta_{n}$ and $\beta_{n m}$ of Ru-PZn with $S_{v}=0.82$ and $\Gamma=0.099 \mathrm{eV}$. The open squares are measured $\beta_{\lambda}$ values at 800,1064 , and $1300 \mathrm{~nm}$ in $\mathrm{CH}_{3} \mathrm{CN}$ solvent. ${ }^{24}$ The irradiation wavelength ranges are divided into three regions (I, II, and III).

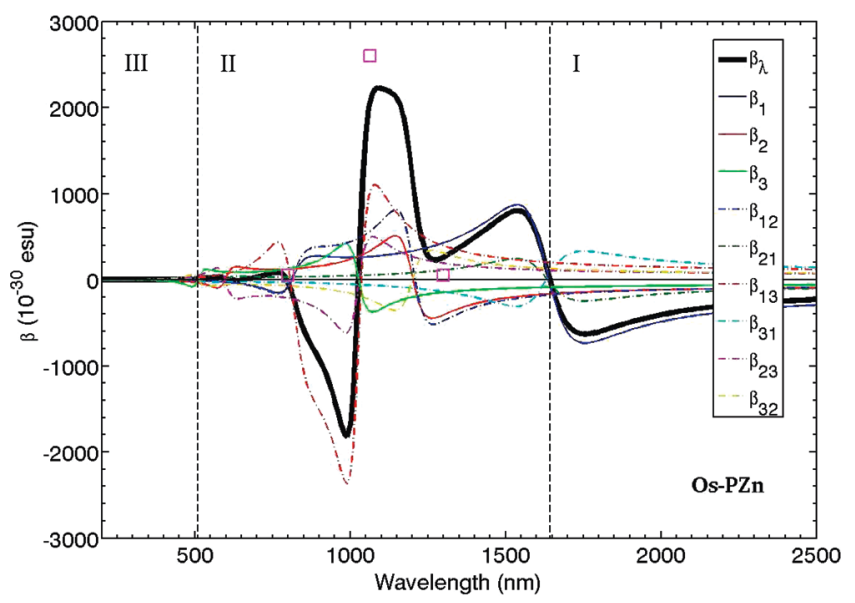

Figure 9. Computed $\beta_{\lambda}, \beta_{n}$, and $\beta_{n m}$ for Os-PZn with $S_{v}=0.83$ and $\Gamma=0.06 \mathrm{eV}$. The open squares are measured $\beta_{\lambda}$ values at 800,1064 , and $1300 \mathrm{~nm}$ in $\mathrm{CH}_{3} \mathrm{CN}$ solvent. ${ }^{24}$ The irradiation wavelength ranges are divided into three regions (I, II, and III).

TABLE 4: Comparisons of the Calculated and Measured Molecular Hyperpolarizabilities $\left(\mathbf{1 0}^{-30}\right.$ esu) for Ru-PZn and Os-PZn in $\mathrm{CH}_{3} \mathrm{CN}$ Solvent ${ }^{24,73 a}$

\begin{tabular}{llllll}
\hline & & $\beta_{800}$ & \multicolumn{1}{c}{$\beta_{1064}$} & \multicolumn{1}{c}{$\beta_{1300}$} & \multicolumn{1}{c}{$\beta_{1500}$} \\
\hline $\mathbf{R u}-\mathbf{P Z n}^{b}$ & calcd & 222 & 1542 & 3796 & 3640 \\
& & $(186)^{d}$ & $(1432)^{d}$ & $(3473)^{d}$ & $(740)^{d}$ \\
& exptl & $<50$ & 2100 & 5100 & $550 \pm 200$ \\
Os-PZn $^{c}$ & calcd & 54 & 2074 & 260 & 749 \\
& exptl & $<50$ & 2600 & $<50$ & $230 \pm 115$
\end{tabular}

${ }^{a} \beta_{800}$ and $\beta_{1300}$ are used to fit $S_{v}$ and $\Gamma$. $\beta_{1064}$ and $\beta_{1500}$ are predicted. The $\beta_{\lambda}$ values in parentheses for $\mathbf{R u}-\mathbf{P Z n}$ are calculated with the adjusted transition energy $\left(E_{01}=1.67 \mathrm{eV}\right) .{ }^{b} S_{v}=0.82, \Gamma$ $=0.099 \mathrm{eV} .{ }^{c} S_{v}=0.83, \Gamma=0.06 \mathrm{eV} .{ }^{d} \beta_{1500}$ was calculated with $E_{01}=1.67 \mathrm{eV}$.

mophore. The calculated $\beta_{\lambda}$ values in Table 4 agree well with the experimental data. The large differences between $\beta_{800}$ and $\beta_{1300}$ values for $\mathbf{R u}-\mathbf{P Z n}$ and Os-PZn were used to fit the spectral shift and half-width parameters and to choose the relevant solution of eq 16 . The predicted $\beta_{1064}$ values (Table 4) agree with the experimental $\beta_{1064}$ values (error $<26 \%$ ). In addition, the fitted shift factors for Ru-PZn and Os-PZn are very similar (0.83 and 0.82 , respectively). We suspect that the similar shift factors are related to the structural similarity of the supermolecules.
Two-Level and Three-Level Contributions to $\boldsymbol{\beta}_{\lambda}$ as a Function of Wavelength. To explain the unusual observed oscillation of the measured $\beta_{\lambda}$ values at 800, 1064, and 1300 nm, we compare $\beta_{\lambda}$ spectra for Ru-PZn and Os-PZn and the nine components of $\beta_{x x x}$ (Figures 8 and 9): $\beta_{1}$ is the two-level $\beta_{\lambda}$ contribution from the $0 \rightarrow Q_{x} \rightarrow Q_{x} \rightarrow 0$ transitions; $\beta_{2}$ is the two-level $\beta_{\lambda}$ contribution from the $0 \rightarrow$ MLCT $\rightarrow$ MLCT $\rightarrow 0$ transitions; $\beta_{3}$ is the two-level $\beta_{\lambda}$ contribution from the 0 $\rightarrow B_{x} \rightarrow B_{x} \rightarrow 0$ transitions; $\beta_{12}$ is the three-level $\beta_{\lambda}$ contribution from the $0 \rightarrow Q_{x} \rightarrow$ MLCT $\rightarrow 0$ transitions; $\beta_{21}$ is the threelevel $\beta_{\lambda}$ contribution from the $0 \rightarrow$ MLCT $\rightarrow Q_{x} \rightarrow 0$ transitions; $\beta_{13}$ is the three-level $\beta_{\lambda}$ contribution from the $0 \rightarrow Q_{x} \rightarrow B_{x} \rightarrow$ 0 transitions; $\beta_{31}$ is the three-level $\beta_{\lambda}$ contribution from the 0 $\rightarrow B_{x} \rightarrow Q_{x} \rightarrow 0$ transitions; $\beta_{23}$ is the three-level $\beta_{\lambda}$ contribution from the $0 \rightarrow$ MLCT $\rightarrow B_{x} \rightarrow 0$ transitions; $\beta_{32}$ is the threelevel $\beta_{\lambda}$ contribution from the $0 \rightarrow B_{x} \rightarrow$ MLCT $\rightarrow 0$ transitions. The above notation describing the $\beta_{\lambda}$ contributions from various transitions denotes only the dominant component of the observed absorptive oscillator strength for these manifolds, as it is known that $B_{x}, Q_{x}$, and MLCT states are strongly mixed in M-PZn chromophores. Since $D_{n m}$ for each $\beta_{n}$ or $\beta_{m n}$ has similar wavelength dependence to that described for the two-band (D-PZn-A) case where the linear spectrum features two absorption manifolds with charge-resonance character, the wavelength dependence of the $D_{n m}$ values for M-PZn are shown in Figures S5 and S6 of Supporting Information.

As for D-PZn-A, for each $\beta_{n}$ or $\beta_{n m}$ function, the requisite one-photon and two-photon resonances appear at $\omega_{1}=\omega_{2}=$ $\omega_{n 0}\left(\right.$ or $\left.\lambda_{1}=\lambda_{2}=\lambda_{n 0}\right)$ and $\omega_{1}=\omega_{2}=\omega_{m 0} / 2$ (or $\lambda_{1}=\lambda_{2}=$ $\left.2 \lambda_{m 0}\right)$, respectively. By reference to the positions of these two resonances, the irradiation wavelength ranges can be divided into three regions (I, II, and III shown in Figures 3, 8, and 9) to analyze the two-level and three-level contributions to $\beta_{\lambda}$.

Region I includes wavelengths longer than the two-photon resonant regime for the low-energy $Q_{x}$-state derived transition manifold, where two-level contributions dominate. In particular, at wavelengths much longer than $2000 \mathrm{~nm}, \beta_{\lambda}$ is dominated by $\beta_{1}$ (the two-level contribution of the $Q_{x}$-derived supermolecular charge-resonance state) because the magnitude of $D_{11}$ exceeds all other $D_{m n}$ terms as the incident frequencies $\omega_{1}$ and $\omega_{2}$ approach zero (eq 9). This suggests that the TKS-SOS formulation can be interpreted using the two-state model (eq 4) when the wavelength is sufficiently long. ${ }^{51,54,55}$ This may also justify the use of only the two-state model for explaining the variation in observed $\beta_{1300}$ values with changes in the $Q$-state transition energy among a small series of push-pull porphyrin-derived chromophores with varying donor and/or acceptor moieties. ${ }^{26,27}$

Region II ranges from the two-photon resonant regime of the $Q_{x}$-derived supermolecular charge-resonance manifold of states to the one-photon resonant wavelength regime for the analogous $B_{x}$-state derived manifold. In this spectral region, both the two-level $\left(\beta_{n}\right)$ and three-level contributions $\left(\beta_{n m}\right)$ need to be considered in order to compute the M-PZn $\beta_{\lambda}$ spectrum. Generally, $\beta_{n}$ or $\beta_{n m}$ can dominate when $\omega_{1}$ is close to $\omega_{n 0}$ (the one-photon resonance frequency), or when $\omega_{1}+\omega_{2}$ is close to $\omega_{m 0}$ (the two-photon resonance frequency). In particular, when $n \neq m$, both $\omega_{m 0}-\omega_{1}-\omega_{2}$ and $\omega_{n 0}-\omega_{1}$ can be small, which causes the contributions from three-level terms to be significant. As shown in Figures 8 and 9, three-level contributions are critical between 400 and $1200 \mathrm{~nm}$ for Ru-PZn and Os-PZn.

Region III includes wavelengths shorter than the $B_{x}$-derived manifold of states, where all of the two-level and three-level contributions are small. Each contribution approaches zero rapidly because the $\beta_{n m}$ denominators are very large when the 
incident wavelength is far from the one- and two-photon resonances, e.g., $<700 \mathrm{~nm}$. Transitions in the shorter wavelength window are also expected to have more limited CT character (compared to transitions in the longer wavelength regime) and, therefore, to make small contributions to the hyperpolarizability.

The predicted frequency dispersion features of each of these three spectral regions explain the unusual oscillatory dependence of $\beta_{\lambda}$ found for the M-PZn chromophores at 800, 1064, and $1300 \mathrm{~nm}$. The theory provides important insights into the relative magnitudes of the hyperpolarizabilities of these chromophores at other wavelengths, making explicit testable predictions at yet unmeasured but accessible wavelengths, such as $1500 \mathrm{~nm}$ (e.g., we predict $\beta_{1500}$ of $\sim 3,600 \times 10^{-30}$ esu for Ru-PZn and $\sim 800$ $\times 10^{-30}$ esu for Os-PZn). At $800 \mathrm{~nm}$, in region II, the measured $\beta_{\lambda}$ values of Ru-PZn and Os-PZn are very small for two reasons: the magnitude of each $\beta_{n}$ or $\beta_{n m}$ at $800 \mathrm{~nm}$ is small (the denominators in eq 7 are large) and the $\beta_{n}$ and $\beta_{n m}$ components of $\beta_{\lambda}$ have different signs. For Ru-PZn, the twolevel contribution $\left(\beta_{1}\right)$ cancels with the other two-level contributions, $\beta_{2}$ and $\beta_{3}$. This cancellation of $B_{x^{-}}$and $Q_{x}$-state derived contributions to the observed hyperpolarizability is consistent with the qualitative analysis originally used to explain the observed frequency dispersion of $\beta_{\lambda}$ in $\mathbf{M}$-PZn chromophores. ${ }^{24}$ Interestingly, this theoretical analysis also shows that the primary three-level contributions, $\beta_{23}$ and $\beta_{32}$, cancel effectively as well with $\beta_{13}$ and $\beta_{12}$.

At $1064 \mathrm{~nm}$, in region II, the $\beta_{\lambda}$ values are generally large for Ru-PZn and Os-PZn because most of the $\beta_{\lambda}$ components, including the two-level and three-level contributions, are large in magnitude. For Os-PZn, the $\beta_{13}$ value is exceptionally large compared to the other components because the transition dipole moment between $Q_{x^{-}}$and $B_{x}$-derived manifolds are dominant among the excited state transition dipoles. This is consistent with experiments that show that $\beta_{1064}$ for Os-PZn increases dramatically from near zero at $800 \mathrm{~nm}$ to $2600 \times 10^{-30}$ esu at $1064 \mathrm{~nm}$. In contrast, for Ru-PZn, the transition dipole moment between the (terpyridyl)metal MLCT- and PZn $B_{x}$-derived manifolds is the largest among the excited states, which makes the corresponding three-level contributions $\left(\beta_{32}\right.$ and $\left.\beta_{23}\right)$ large in magnitude. Note, however, that the two-level contributions $\left(\beta_{1}\right.$ and $\left.\beta_{2}\right)$ are largely derived from the large dipole moment differences: $\bar{x}_{11}=-12.58$ au and $\bar{x}_{22}=17.05 \mathrm{au}$. Therefore, the combined effect of large $\beta$ components for $\mathbf{R u}-\mathbf{P Z n}$ lead to a large observed $\beta_{\lambda}\left(2,100 \times 10^{-30}\right.$ esu in experiment $)$ value at $1064 \mathrm{~nm}$, where $\beta_{1}$ and $\beta_{32}$ make positive contributions, while $\beta_{2}$ and $\beta_{23}$ make negative contributions with much larger absolute values than $\beta_{1}$ and $\beta_{32}$.

At $1300 \mathrm{~nm}$ (region I) the dramatic $\beta_{\lambda}$ value differences for Ru-PZn and Os-PZn are caused largely by their two-level $\beta_{\lambda}$ contributions. For Ru-PZn, the measured $\beta_{1300}\left(5,100 \times 10^{-30}\right.$ esu) value is very large. Its exceptional magnitude arises from the two-level contributions $\beta_{1}$ and $\beta_{2}$ that result from large CT dipole moment differences $\bar{x}_{11}=-12.58$ au and $\bar{x}_{22}=17.05$ au. $\beta_{11}$ and $\beta_{22}$ also have the same sign. The three-level contributions for Ru-PZn at $1300 \mathrm{~nm}$ are negligible. In contrast, for Os-PZn, all of the two-level contributions that originate in the $B_{x^{-}}$and $Q_{x}$-derived supermolecular charge-resonance manifolds are minor because the values of $\bar{x}_{11}, \bar{x}_{22}$, and $\bar{x}_{33}$ are small, and $\beta_{1}$ is canceled by $\beta_{2}$ and $\beta_{3}$. Meanwhile, the three-level contributions, $\beta_{12}$ and $\beta_{23}$, are canceled by the other three-level $\beta_{n m}$ values.

This analysis shows that for the Ru-PZn and Os-PZn chromophores the unusual observed frequency dependence of the molecular hyperpolarizability cannot be explained quanti- tatively without including three-level contributions that arise from the transition dipoles among $Q_{x^{-}}$, MLCT-, and $B_{x}$-derived manifolds. It is important to underscore that the similarity of the observed linear absorption spectra for Ru-PZn and Os-PZn does not guarantee similar transition dipoles among the excited states that display charge-resonance character. As such, $\beta_{\lambda}$ values for species with similar linear absorption spectra can be quite different. The unusually large calculated $x_{23}$ value $\left(x_{23}=11.50\right.$ au) of Ru-PZn suggests strong interactions among MLCT and $B$ states. ${ }^{17,24,74}$ In contrast, for Os-PZn, the interaction between the MLCT- and $B_{x}$-derived manifolds is much weaker $\left(x_{23}=\right.$ $-3.17 \mathrm{au}) .^{28}$

Further $\beta_{\lambda}$ tests for Ru-PZn and Os-PZn at 1500 nm. To further test the predicted $\beta_{\lambda}$ spectra, we recently measured $\beta_{\lambda}$ values at $1500 \mathrm{~nm}$ for Ru-PZn and Os-PZn. ${ }^{73}$ The measured and predicted $\beta_{1500}$ values are shown in the last column of Table 4. For Os-PZn, our model predicts a $\beta_{1500}$ value that is consistent with the experimental value and thus successfully mirrors the trend in $\beta_{\lambda}$ at 800, 1064, 1300, and $1500 \mathrm{~nm}$ (i.e., $\beta_{1064}>\beta_{1500}$ $>\beta_{1300} \simeq \beta_{800}$ ). For Ru-PZn, the predicted $\beta_{1500}$ is overestimated by about 5 -fold. This error in $\beta_{1500}$ is caused by the two-photon resonance effects. As shown in Figure 8 , the $\beta_{\lambda}$ spectrum for Ru-PZn near $1500 \mathrm{~nm}$ is dominated by the contribution of $\beta_{1}$ which has a resonance at $1560 \mathrm{~nm}$. In the vicinity of this resonance, minor shifts in the excitation wavelength or minor error in the estimated transition energy can cause large changes in $\beta_{\lambda}$. For example, if $E_{01}$ of $\mathbf{R u}-\mathbf{P Z n}$ is shifted slightly from $1.59 \mathrm{eV}(780 \mathrm{~nm})$ to $1.67 \mathrm{eV}(743 \mathrm{~nm})$, say, by a possible $5 \%$ $E_{01}$ estimation error, the predicted $\beta_{1500}$ value drops from 3,640 $\times 10^{-30}$ esu to $740 \times 10^{-30} \mathrm{esu}$, which is close to the experimental value of $(550 \pm 200) \times 10^{-30}$ esu. Thus, the prediction of $\beta_{\lambda}$ values very near resonance (e.g., $\beta_{1500}$ for $\mathbf{R u}$ PZn) depends critically on accurate estimation of the transition energy and can also be influenced by the errors in the transition dipole moments and of first excited state lifetimes. Even though large error in $\beta_{\lambda}$ values may arise very close to resonance, the predicted $\beta_{\lambda}$ values further from resonance show much smaller errors. For example, as noted in Table 4 (the predicted $\beta_{\lambda}$ values in parentheses), a possible $5 \%$ error of $E_{01}$ causes only $\sim 10 \%$ changes for $\beta_{\lambda}$ at 800,1064 , and $1300 \mathrm{~nm}$. Therefore, our simple TKS-SOS model predicts the overall structure of the $\beta_{\lambda}$ spectrum accurately, and this empirical approach also predicts specific values of $\beta_{\lambda}$ quantitatively, when they do not lie too close to electronic resonances.

\section{Conclusions}

For the first time, we have predicted the frequency dispersion of hyperpolarizabilities using a SOS expression with both twoand three-level contributions by employing measured linear absorption data and generalized TKS rules. In contrast, the conventional two-state $\beta$-approximation exclusively uses a single excited state to qualitatively describe the hyperpolarizability. Inclusion of both multiple excited states and three-level terms, the TKS-SOS approach developed here produces frequencydependent $\beta$ spectra in quantitative agreement with the measured experimental values. While earlier studies have argued that relatively few effective excited states were required to describe off-resonance $\beta$ values, this is the first study to successfully use a relatively small number of effective excited states to describe the full frequency dispersion of $\beta$. Predicting the dynamic $\beta$ could prove of use for the design and synthesis of novel next-generation NLO materials.

The generalized TKS-SOS method was applied to an archetypal porphyrin-based, high $\beta_{\lambda}$ chromophore, D-PZn-A, ${ }^{19,21,23}$ 
with two dominant absorption manifolds that possess chargeresonance character; this analysis demonstrated the importance of two- and three-level transitions to the magnitude of $\beta_{\lambda}$, and provided quantitative agreement with previously acquired experimental data. These methods were next used to interrogate the origins of the nonlinear responses of electronically complex chromophores that feature coupled-oscillator photophysics and display oscillatory dependences of the molecular hyperpolarizability with incident wavelength. It was established that M-PZn chromophores, where (porphinato)zinc(II)(PZn) and metal(II)polypyridyl (M) units are connected via an ethyne unit that aligns the high oscillator strength transition dipoles of these components in a head-to-tail arrangement, can be designed such that $B$ - and $Q$-state-derived $\beta_{0}$ values can have the same or opposite sign. This effect, coupled with the fact that the sign of the resonance enhancement factor is frequency dependent, gives rise to NLO chromophores that manifest unusual wavelengthdependent hyperpolarizabilities. ${ }^{24} \mathbf{R u}-\mathbf{P Z n}$ and $\mathbf{O s - P Z n}$ are a particularly interesting pair of such chromophores because of their similar linear-absorption spectra but dramatically different dynamic hyperpolarizabilities. This is underscored by the fact that while Ru-PZn features an extraordinarily large hyperpolarizability at telecommunication relevant wavelengths the analogous response for Os-PZn is too small to measure. ${ }^{24}$ The generalized TKS-SOS method accurately predicted the experimental $\beta_{\lambda}$ dependences for Ru-PZn and Os-PZn at 800, 1064, and $1300 \mathrm{~nm}$, and for Os-PZn at $1500 \mathrm{~nm}$; we note that even though a significant error is found in the predicted $\beta_{\lambda}$ value at $1500 \mathrm{~nm}$ for Ru-PZn, this derives solely from a resonance effect. It is important to emphasize, however, that the overall shape of the predicted $\beta_{\lambda}$ spectrum is meaningful, especially for the quantitative prediction of $\beta_{\lambda}$ values off resonance.

In summary, generalized TKS analysis provides a quantitative strategy to link measured linear optical absorption spectra with the frequency-dependent nonlinear optical response. Further, this work underscores that, although structurally related NLO chromophores may possess similar linear absorption spectra, dramatically different frequency-dependent hyperpolarizabilities can be manifested when strongly coupled CT transitions are present along with corresponding three-level contributions that result from excited state-to-excited state transition dipoles between multiple charge-resonance states. Finally, we believe that this hybrid approach to predicting frequency-dependent hyperpolarizabilities will assist in tailoring NLO chromophoresand interpreting their response-for wavelength-specific applications because: (i) supermolecules with multiple CT transitions interacting as coupled oscillators provide a means to elaborate highly hyperpolarizable chromophores, (ii) NLO devices operate at finite wavelengths with nontrivial frequencydependent responses, and (iii) measured $\beta_{\lambda}$ values and their theoretical interpretations provide a productive link among chromophore structure, absorption spectra, frequency-dependent hyperpolarizabilities, and the electronic states that dominate the nonlinear response.

Acknowledgment. Support from the DARPA project "Predicting Real Optimized Materials" through ARO is gratefully acknowledged (W911NF-04-1-0243). D.N.B. thanks the Keck and NEDO Foundations for support of computational infrastructure, and NSF Grant CHE-0718043 for additional support. W.Y. and M.J.T. acknowledge, respectively, partial support of the National Science Foundation and the Department of Energy (DE-FG02-04ER46156). M.J.T. is grateful to the Francqui Foundation (Belgium) and VLAC (Vlaams Academisch Centrum), Centre for Advanced Studies of the Royal Flemish
Academy of Belgium for Science and the Arts, for research fellowships. K.C. thanks the Flemish Fund for Scientific Research (FWO G.0312.08) for financial support. I.A. acknowledges a postdoctoral fellowship from the Fund for Scientific Research, Flanders (FWO-V).

Supporting Information Available: All experimental and theoretical evidence to support our current work and figures showing the wavelength dependence of $D_{m n}$ for $\mathbf{R u}-\mathbf{P Z n}$ and Os-PZn. This material is available free of charge via the Internet at http://pubs.acs.org/.

\section{References and Notes}

(1) Marder, S. R.; Sohn, J. E.; Stucky, G. D. Materials for Nonlinear Optics: Chemical Perspectives; American Chemical Society: Washington, DC, 1991; Vol. 455.

(2) Marder, S. R.; Beratan, D. N.; Cheng, L. T. Science 1991, 252, 103.

(3) Dakhnovskii, Y. I.; Pronin, K. A. Synth. Met. 1993, 54, 295.

(4) Pronin, K. A.; Dakhnovskii, Y. I.; Bandrauk, A. D. Synth. Met. 1993, $57,3907$.

(5) Burland, D. M. Chem. Rev. 1994, 94, 1.

(6) DiBella, S.; Lanza, G.; Fragala, I.; Yitzchaik, S.; Ratner, M. A.; Marks, T. J. J. Am. Chem. Soc. 1997, 119, 3003.

(7) Albert, I. D. L.; Marks, T. J.; Ratner, M. A. J. Am. Chem. Soc. 1997, 119,6575

(8) Keinan, S.; Zojer, E.; Bredas, J. L.; Ratner, M. A.; Marks, T. J. THEOCHEM 2003, 633, 227.

(9) Keinan, S.; Therien, M. J.; Beratan, D. N.; Yang, W. J. Phys. Chem. A 2008, 112, 12203 .

(10) Hu, X. Q.; Beratan, D. N.; Yang, W. T. J. Chem. Phys. 2008, 129,

(11) Xiao, D. Q.; Bulat, F. A.; Yang, W. T.; Beratan, D. N. Nano Lett. 2008, 8,2814

(12) Xiao, D. Q.; Yang, W. T.; Beratan, D. N. J. Chem. Phys. 2008, 129,044106

(13) Kuzyk, M. G. IEEE J. Sel. Top. Quantum Electron. 2001, 7, 774.

(14) Kuzyk, M. G. Phys. Rev. A 2005, 72, 053819.

(15) Tripathy, K.; Moreno, J. P.; Kuzyk, M. G.; Coe, B. J.; Clays, K.; Kelley, A. M. J. Chem. Phys. 2004, 121, 7932.

(16) Zhou, J. F.; Kuzyk, M. G. J. Phys. Chem. C 2008, 112, 7978.

(17) Duncan, T. V.; Song, K.; Hung, S. T.; Miloradovic, I.; Nayak, A.; Persoons, A.; Verbiest, T.; Therien, M. J.; Clays, K. Angew. Chem., Int. Ed. 2008, 47, 2978.

(18) Meyers, F.; Marder, S. R.; Pierce, B. M.; Brédas, J. L. J. Am. Chem. Soc. 1994, 116, 10703.

(19) Karki, L.; Vance, F. W.; Hupp, J. T.; LeCours, S. M.; Therien, M. J. J. Am. Chem. Soc. 1998, 120, 2606.

(20) LeCours, S. M.; DiMagno, S. G.; Therien, M. J. J. Am. Chem. Soc. 1996, 118,11854 .

(21) LeCours, S. M.; Guan, H. W.; DiMagno, S. G.; Wang, C. H.; Therien, M. J. J. Am. Chem. Soc. 1996, 118, 1497.

(22) Marder, S. R.; Cheng, L.-T.; Tiemann, B. G.; Friedli, A. C.; Blanchard-Desce, M.; Perry, J. W.; J., S. Science 1994, 263, 511.

(23) Priyadarshy, S.; Therien, M. J.; Beratan, D. N. J. Am. Chem. Soc. 1996, 118, 1504

(24) Uyeda, H. T.; Zhao, Y. X.; Wostyn, K.; Asselberghs, I.; Clays, K.; Persoons, A.; Therien, M. J. J. Am. Chem. Soc. 2002, 124, 13806.

(25) Kang, H.; Facchetti, A.; Zhu, P. W.; Jiang, H.; Yang, Y.; Cariati, E.; Righetto, S.; Ugo, R.; Zuccaccia, C.; Macchioni, A.; Stern, C. L.; Liu, Z. F.; Ho, S. T.; Marks, T. J. Angew. Chem., Int. Ed. 2005, 44, 7922.

(26) Zhang, T.-G.; Zhao, Y. X.; Asselberghs, I.; Persoons, A.; Clays, K.; Therien, M. J. J. Am. Chem. Soc. 2005, 127, 9710.

(27) Zhang, T.-G.; Zhao, Y. X.; Song, K.; Asselberghs, I.; Persoons, A.; Clays, K.; Therien, M. J. Inorg. Chem. 2006, 45, 9703.

(28) Duncan, T. V.; Ishizuka, T.; Therien, M. J. J. Am. Chem. Soc. 2007, $129,9691$.

(29) Shediac, R.; Gray, M. H. B.; Uyeda, H. T.; Johnson, R. C.; Hupp, J. T.; Angiolillo, P. J.; Therien, M. J. J. Am. Chem. Soc. 2000, 122, 7017. (30) Rubtsov, I. V.; Susumu, K.; Rubtsov, G. I.; Therien, M. J. J. Am. Chem. Soc. 2003, 125, 2687.

(31) Susumu, K.; Therien, M. J. J. Am. Chem. Soc. 2002, 124, 8550.

(32) Susumu, K.; Duncan, T. V.; Therien, M. J. J. Am. Chem. Soc. 2005, 127,5186 .

(33) Duncan, T. V.; Susumu, K.; Sinks, L. E.; Therien, M. J. J. Am. Chem. Soc. 2006, 128, 9000.

(34) Thorley, K. J.; Hales, J. M.; Anderson, H. L.; Perry, J. W. Angew. Chem., Int. Ed. 2008, 47, 7095 . 
(35) Drobizhev, M.; Stepanenko, Y.; Dzenis, Y.; Karotki, A.; Rebane, A.; Taylor, P. N.; Anderson, H. L. J. Phys. Chem. B 2005, 109, 7223.

(36) Screen, T. E. O.; Thorne, J. R. G.; Denning, R. G.; Bucknell, D. G.; Anderson, H. L. J. Am. Chem. Soc. 2002, 124, 9712.

(37) (a) Reeve, J. E.; Collins, H. A.; De Mey, K.; Kohl, M. M.; Thorley, K. J.; Paulsen, O.; Clays, K.; Anderson, H. L. J. Am. Chem. Soc. 2009, 131, 2758. (b) Therien, M. J. Nature 2009, 458, 716.

(38) Champagne, B.; Perpete, E. A.; Jacquemin, D.; van Gisbergen, S. J. A.; Baerends, E. J.; Soubra-Ghaoui, C.; Robins, K. A.; Kirtman, B. J. Phys. Chem. A 2000, 104, 4755.

(39) Tretiak, S.; Chernyak, V. J. Chem. Phys. 2003, 119, 8809.

(40) Tretiak, S.; Mukamel, S. Chem. Rev. 2002, 102, 3171.

(41) Kirtman, B.; Champagne, B. Int. Rev. Phys. Chem. 1997, 16, 389

(42) Jamorski, C.; Casida, M. E.; Salahub, D. R. J. Chem. Phys. 1996, 104, 5134.

(43) van Gisbergen, S. J. A.; Snijders, J. G.; Baerends, E. J. J. Chem. Phys. 1998, 109, 10644.

(44) van Gisbergen, S. J. A.; Snijders, J. G.; Baerends, E. J. J. Chem. Phys. 1998, 109, 10657.

(45) Champagne, B.; Perpete, E. A.; van Gisbergen, S. J. A.; Baerends, E. J.; Snijders, J. G.; Soubra-Ghaoui, C.; Robins, K. A.; Kirtman, B. J. Chem. Phys. 1998, 109, 10489.

(46) van Gisbergen, S. J. A.; Kootstra, F.; Schipper, P. R. T.; Gritsenko,

O. V.; Snijders, J. G.; Baerends, E. J. Phys. Rev. A 1998, 57, 2556.

(47) Karna, S. P.; Prasad, P. N.; Dupuis, M. J. Chem. Phys. 1991, 94, 1171

(48) Miura, M.; Aoki, Y.; Champagne, B. J. Chem. Phys. 2007, 127.

(49) Kelley, A. M. J. Opt. Soc. Am. B 2002, 19, 1890.

(50) Rebane, A.; Makarov, N. S.; Drobizhev, M.; Spangler, B.; Tarter, E. S.; Reeves, B. D.; Spangler, C. W.; Meng, F. Q.; Suo, Z. Y. J. Phys. Chem. C 2008, 112, 7997 .

(51) Boyd, R. W. Nonlinear Optics; Academic Press: New York, 1992.

(52) Kanis, D. R.; Lacroix, P. G.; Ratner, M. A.; Marks, T. J. J. Am. Chem. Soc. 1994, 116, 10089.

(53) Kanis, D. R.; Ratner, M. A.; Marks, T. J. Chem. Rev. 1994, 94, 195.
(54) Oudar, J. L.; Chemla, D. S. J. Chem. Phys. 1977, 66, 2664.

(55) Oudar, J. L. J. Chem. Phys. 1977, 67, 446.

(56) Risser, S. M.; Beratan, D. N.; Marder, S. R. J. Am. Chem. Soc. 1993, 115, 7719 .

(57) Albert, I. D. L.; Marks, T. J.; Ratner, M. A. J. Am. Chem. Soc. 1998, $120,11174$.

(58) Kanis, D. R.; Lacroix, P. G.; Ratner, M. A.; Marks, T. J. J. Am. Chem. Soc. 1994, 116, 10089.

(59) Shankar, R. Principles of Quantum Mechanics; Plenum Press: New York, 1980.

(60) Kuzyk, M. G. Phys. Rev. Lett. 2000, 85, 1218.

(61) Kuzyk, M. G. Phys. Rev. Lett. 2005, 95, 109402.

(62) Kuzyk, M. G. J. Nonlinear Opt. Phys. Mater. 2006, 15, 77. 2891

(64) Nandi, P. K.; Panja, N.; Ghanty, T. K.; Kar, T. J. Phys. Chem. A 2009, 113, 2623.

(65) Kuzyk, M. G. J. Chem. Phys. 2006, 125, 154108.

(66) Perez-Moreno, J. Theoretical and experimental characterization of the first hyperpolarizability, PhD Thesis, Katholieke Universiteit, 2007.

67) Gilber, A.; Baggott, J. Essentials of Molecular Photochemistry; Blackwell Scientific Publications: Oxford, 1991.

(68) Baxter, C. J. Phys. B 1992, 25, L589.

(69) Champagne, B.; Kirtman, B. Phys. Rev. Lett. 2005, 95, 1.

(70) Champagne, B.; Kirtman, B. J. Chem. Phys. 2006, 125, 024101.

(71) Kuzyk, M. G. Phys. Rev. Lett. 2003, 90, 1

(72) Albota, M.; Beljonne, D.; Bredas, J. L.; Ehrlich, J. E.; Fu, J. Y.; Heikal, A. A.; Hess, S. E.; Kogej, T.; Levin, M. D.; Marder, S. R.; McCordMaughon, D.; Perry, J. W.; Rockel, H.; Rumi, M.; Subramaniam, C.; Webb, W. W.; Wu, X. L.; Xu, C. Science 1998, 281, 1653.

(73) Experimental data from the Clays and Therien groups.

(74) Duncan, T. V.; Rubtsov, I. V.; Uyeda, H. T.; Therien, M. J. J. Am. Chem. Soc. 2004, 126, 9474.

JP911556X 